\title{
UBICACIÓN ÓPTIMA DE RECONECTADORES EN SISTEMAS DE DISTRIBUCIÓN PARA MINIMIZAR LA FRECUENCIA MEDIA DE INTERRUPCIÓN
}

\section{OPTIMAL LOCATION OF RECLOSERS IN DISTRIBUTION NETWORKS IN ORDER TO MINIMIZE THE AVERAGE INTERRUPTION FREQUENCY}

\author{
Jerson Reyes S. ${ }^{1} \quad$ Mariano Morales F. $^{2} \quad$ Luis García-Santander $^{3} \quad$ Jorge E. Pezoa $^{4}$ \\ Recibido 5 de agosto de 2008, aceptado 17 de abril de 2009 \\ Received: August 5, 2008 Accepted: April 17, 2009
}

\begin{abstract}
RESUMEN
En este trabajo se presenta una metodología para minimizar la frecuencia de fallas en Sistemas de Distribución radiales considerando una carga uniformemente distribuida. Se utiliza una aproximación lineal para la ubicación de reconectadores. Este trabajo basa su estudio en la minimización del índice de Frecuencia Media de Interrupción por Potencia instalada, el cual es utilizado como parámetro de regulación y clasificación de las compañías distribuidoras chilenas, según la reglamentación eléctrica vigente. Se introduce además el concepto de Factor de Distancia entre línea Troncal y arranque, el cual puede ser utilizado como herramienta de discriminación para acotar el número de reconectadores a instalar en la línea troncal. Además, permite discriminar en base a la data histórica de la línea si un arranque cualquiera debe ser evaluado económicamente, como un nuevo alimentador, de forma tal que el índice Frecuencia Media de Interrupción por Potencia instalada sea minimizado.
\end{abstract}

Palabras clave: Red de distribución, confiabilidad, ubicación óptima, reconectadores, frecuencia media de interrupción.

\begin{abstract}
This paper presents a framework for minimizing the average interruption frequency in radial Distribution Systems with uniformly distributed loads. Here, the position of the reclosers has been modeled using a linear approximation. The objective of this paper is to minimize the average interruption frequency per installed power. According to the current electric regulation in Chile, this index is the metric used to regulate and classify the performance of utilities. In the analysis a novel concept of Distance Factor between the main feeder and a branch feeder was introduced. This concept can be utilized as a theoretical limit for the number of switches to install on the main feeder. In addition, based upon the service history of the line, the distance factor determines if a certain line must be economically assessed like a new feeder so that the aforementioned performance index of average interruption frequency per installed power is minimized.
\end{abstract}

Keywords: Distribution network, reliability, optimal allocation, reclosers, average interruption frequency.

\section{INTRODUCCIÓN}

En mercados eléctricos no-regulados los clientes tienen un amplio rango de libertad relacionado con la elección de quién le suministra la energía. La figura 1 permite observar cómo algunos clientes están dispuestos a pagar más por mejor calidad, mientras otros estarían dispuestos a aceptar menor calidad a cambio de menores precios. En estos mercados los agentes se ubican de modo que satisfacen sus requerimientos individuales a través de una realimentación precio-calidad. Esta realimentación entre suministrador y consumidor no es posible en los sectores monopólicos y por lo tanto el regulador debe crear una estrategia de regulación [1-3].

En Chile la Comisión Nacional de Energía (CNE) y la Superintendencia de Electricidad y Combustible (SEC) han creado un mecanismo de regulación tal que permite medir

1 Comisión Chilena de Energía Nuclear CCHEN, CEN La Reina, Las Condes. Santiago, Chile. E-mail: jereyes@cchen.cl

2 Compañía General de Electricidad CGE, Administración. Concepción, Chile. E-mail: mmoralesf@cgedistribucion.cl

3 Universidad de Concepción. Departamento de Ingeniería Eléctrica. Barrio Universitario s/n. Concepción, Chile. E-mail: luigarci@udec.cl

4 Universidad de Nuevo México. Departamento de Ingeniería Eléctrica y Computación. Albuquerque, NM, USA. E-mail: jpezoa@ece.unm.edu 
en un conjunto de índices de calidad la percepción del cliente con respecto a la calidad del suministro entregado. En materia de confiabilidad, estos índices son similares a: SAIFI, SAIDI y ENS [4-8], que están orientados al cliente. En Chile se han establecido dos tipos de índices de confiabilidad: basados en transformadores y en los KVA suministrados. Estos índices son usados por la empresa distribuidora como señales económicas de la cantidad de energía vendida y además como un indicador del servicio continuo entregado a los clientes. Este índice es utilizado para realizar inversiones de forma de minimizar las pérdidas, evitar multas por calidad de suministro y utilizados para cálculo en el pago de compensaciones a los clientes por tiempo o energía que no se haya suministrado, tal como se muestra en figura 2 .

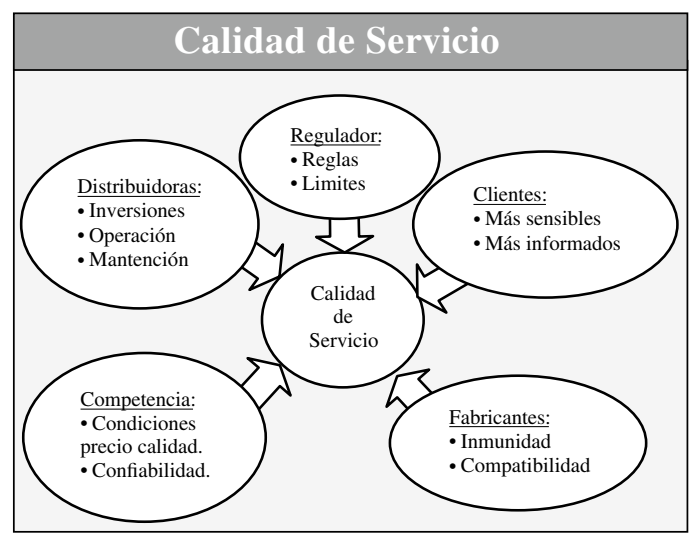

Figura 1. Diagrama de calidad de servicio.

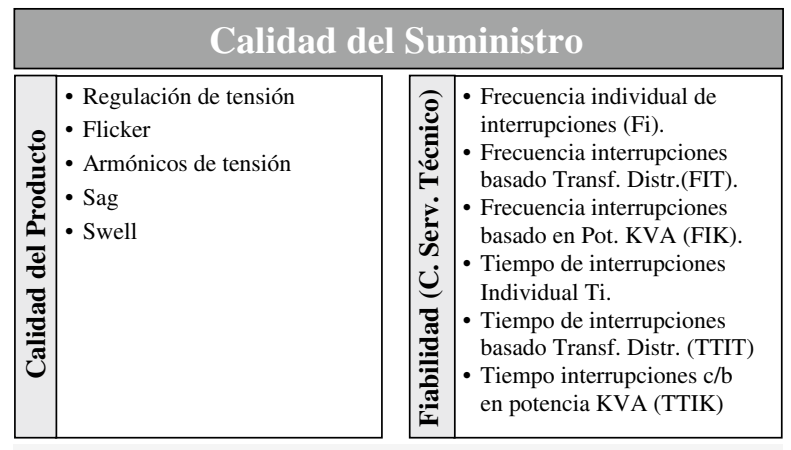

Valores estadísticos garantizados el 95\% del tiempo de una semana

Figura 2. Calidad de suministro.

Para evitar el pago de multas y/o compensaciones tanto al organismo regulador (SEC) como a los propios clientes, las distribuidoras deben invertir en mejoras de la confiabilidad de sus redes, lo que implica en la práctica la incorporación de equipos de protección y/o maniobra o elementos de mayor tecnología como reconectadores. El objetivo principal es minimizar los índices asociados a tiempos y frecuencia (por ejemplo Ti y/o Fi [9]), sin embargo, la inversión a realizar se justificará cuando la eficiencia de la red retribuya en el tiempo las multas por cortes como clientes sin suministro [10-12]. En base a este escenario el objetivo principal en este trabajo de investigación es minimizar el índice asociado a la Frecuencia Media de Interrupción por KVA suministrado (FMIK). Se considera para el método propuesto la ubicación de equipos reconectadores en las líneas troncales del SD y si se cuenta o no con presencia de equipos de protección y/o maniobra en estado normalmente abierto, los cuales permiten una posible reposición del servicio eléctrico a ciertas cargas del SD.

\section{FUNDAMENTACIÓN TEÓRICA}

Los SD operan generalmente en una configuración radial o arborescente. Un alimentador incluye tramos de línea, cables, dispositivos de interrupción, de protección, entre otros. Según esta operación de los alimentadores podemos asumir, desde el punto de la confiabilidad, que todos los elementos están conectados en serie. Cada componente que está presente en el alimentador tiene su propia tasa de fallas definida como en la ecuación (1) siguiente.

$\lambda=\frac{\text { Número_fallas_por_unidad_tiempo }}{\text { Número_componentes_expuestos_falla }}$

En un alimentador de distribución la tasa de falla $\lambda$ total es equivalente a la suma de las tasas de falla de cada elemento (conexión serie) y está directamente relacionada con los aspectos constructivos de la línea y con la agresividad del medio en que se encuentra. Por lo tanto, para mejorar la tasa de falla equivalente del alimentador es necesario usar componentes más confiables cuyo comportamiento se puede describir según la curva de la bañera, presentada en la figura 3.

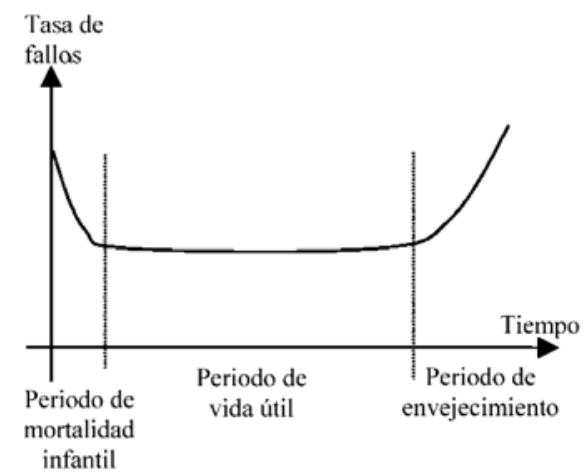

Figura 3. Curva de la bañera.

En Chile el órgano regulador ha establecido, entre otros, los siguientes índices de frecuencia y duración equivalente de las interrupciones. 


$$
F M I K=\frac{\sum_{i=1}^{n} c a r g a \_K V A \_i n t e r r u m p i d a}{T o t a l \_c a r g a \_K V A \_e n \_s i s t e m a}
$$

En el presente trabajo se considera un sistema radial como se presenta en la figura 4 , con una fuente de alimentación principal y un equipo de protección en la cabecera de la troncal. Otro caso de estudio es el que se presenta en la figura 6 , donde la presencia de equipos de protección y/o maniobra en estado normalmente abierto (NA) permiten la alimentación desde otras fuentes o alimentadores. La fuente se considera para este trabajo como siempre disponible y cuya tasa de fallas se considera nula.

Bajo los casos de estudio, las tasas de falla a lo largo de la línea serán conocidas considerando:

- Tasa de falla constante: valor promedio anual, considerando un análisis de la línea dentro del periodo de vida útil (figura 3).

- En función de las estadísticas de fallas por tramo de línea, se establece una curva $\lambda$ (fallas $\cdot \mathrm{km}$ ) que incorpora la tasa de falla por sectores integrando así la influencia de los arranques relevantes de la línea troncal en el análisis.

La tasa de falla conocida y constante es utilizada para simplificar el análisis de la línea, sin embargo, esta no necesariamente es igual a la de los arranques, pero pueden ser integradas si se considera el análisis conforme al segundo punto. Por simplicidad se considera en todos los cálculos posteriores que $\lambda$ es constante, y que cada arranque tiene la misma potencia conectada (KVA).

\section{INFLUENCIA DE LOS ARRANQUES}

Para investigar la influencia de las fallas en arranques en el índice de frecuencia, considere la figura 4 donde un alimentador de longitud $\mathbf{L}(\mathrm{km})$ posee $\boldsymbol{a}_{\boldsymbol{n}}$ arranques, cada uno de ellos con un fusible como protección principal. Consideremos además una longitud de $\mathbf{b} / \mathbf{2}(\mathrm{km})$ por arranque, dos equipos de protección tales como los reconectadores a lo largo de la línea troncal instalados en $\mathbf{x}=\mathbf{0}(\mathbf{k m})$ y $\mathbf{x}=\mathbf{L} / \mathbf{2}(\mathbf{k m})$. Con tasas de falla iguales para cada línea troncal y cada línea de los arranques $\left(\lambda_{\mathrm{T}}=\lambda_{\mathrm{a}}=\lambda\right)$ y con el supuesto que sólo se produce una falla a la vez.

La tabla 1 muestra cómo evoluciona el índice a medida que ocurren fallas en diversas áreas de alimentador.

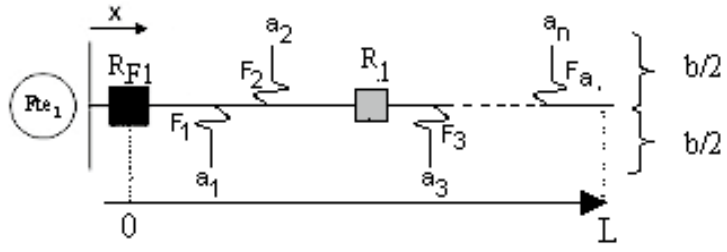

Figura 4. Alimentador de largo L $(\mathrm{km}) \operatorname{con} a_{n}$ arranques.

Estas fallas hacen actuar los equipos de protección presentes en el alimentador (reconectadores $R_{i}$ y Fusibles $F_{i}$ ). La primera columna entrega información de la protección operada al ocurrir una falla; la segunda columna permite visualizar la potencia que se interrumpe al ocurrir la falla; finalmente la tercera columna en función de la frecuencia de falla entrega la frecuencia de interrupciones asociadas a los KVA interrumpidos al operar la protección asociada.

Tabla 1. Tabla de eventos para cálculo del índice de frecuencia.

\begin{tabular}{|c|c|c|}
\hline $\begin{array}{c}\text { Protección } \\
\text { Operada }\end{array}$ & $\begin{array}{c}\text { KVA } \\
\text { Interrumpidos }\end{array}$ & $\begin{array}{c}\text { Frecuencia de } \\
\text { Interrupción } \mathbf{F}_{\mathbf{i}}\end{array}$ \\
\hline $\mathrm{R}_{\mathrm{F} 1}$ & $K V A_{T}$ & $\frac{L \cdot \lambda_{T}}{2} \cdot K V A_{T}$ \\
\hline $\mathrm{R}_{1}$ & $\frac{K V A_{T}}{2}$ & $\frac{L \cdot \lambda_{T}}{4} \cdot K V A_{T}$ \\
\hline $\mathrm{F}_{1}$ & $\frac{K V A_{T}}{a}$ & $\frac{\lambda_{a} \cdot b}{2} \cdot \frac{K V A_{T}}{a}$ \\
\hline$\ldots$ & $\frac{K V A_{T}}{a}$ & $\frac{\lambda_{a} \cdot b}{2} \cdot \frac{K V A_{T}}{a}$ \\
\hline $\mathrm{F}_{\mathrm{a}}$ & & \\
\hline
\end{tabular}

Entonces la frecuencia de interrupción total:

$$
K V A_{\text {Total }}\left(\frac{L \cdot \lambda_{T}}{2}+\frac{L \cdot \lambda_{T}}{4}+\frac{b \cdot \lambda_{a}}{2}\right)
$$

Luego el índice de frecuencia FMIK resultante para el sistema en estudio queda dado por (4).

$$
F M I K_{\text {Total }}=\frac{3 \lambda_{T} \cdot L}{4}+\frac{\lambda_{a} \cdot b}{2}
$$

La expresión (4) muestra que los eventos que afectan la línea troncal tienen mayor importancia en el índice de frecuencia. Por lo que se deduce del resultado anterior que se deben enfocar mayormente los análisis de prevención de fallas en la línea troncal. 
Extrapolando el análisis anterior surge la propuesta de un nuevo índice el cual se denominará Factor de Distancias Troncal-arranque $\boldsymbol{F} \boldsymbol{D}_{\boldsymbol{T a}}$, este factor se obtendrá entre el cuociente del FMIK relacionado a la línea troncal de tasa de falla $\lambda_{T}$, y largo $\mathbf{L}(\mathrm{km})$, con el FMIK relacionado al

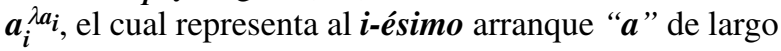
$\boldsymbol{b}(\mathrm{km})$ y tasa de falla $\lambda_{\boldsymbol{a}_{\boldsymbol{i}}}$

$$
\frac{F M I K_{T}}{F M I K_{a}}=F D_{T a} \cdot \frac{L}{b}
$$

Esta expresión (5) permite establecer un criterio de evaluación en función de la frecuencia de falla del arranque bajo análisis y de la troncal. Por lo tanto el $\boldsymbol{F} \boldsymbol{D}_{\boldsymbol{T a}}$ permite evaluar si la influencia de las fallas del arranque analizado es lo suficientemente relevante sobre la línea troncal, es decir, indicará si es necesario evaluar el arranque como una nueva línea troncal. Esto implica una reestructuración de la línea y por ende una nueva ubicación de los equipos reconectadores instalados, de forma de minimizar la frecuencia de fallas FMIK y optimizar así, la inversión a realizar, por lo tanto:

- $\quad$ Si $\boldsymbol{F} \boldsymbol{D}_{T \boldsymbol{T}}=\mathbf{1}$, implica que la influencia en las fallas del arranque bajo estudio es el mismo que el de la troncal, por lo que se sugiere, bajo el posible crecimiento de la densidad de demanda en el arranque en cuestión, considerarlo en el futuro como una nueva troncal.

- Si $\boldsymbol{F} \boldsymbol{D}_{\boldsymbol{T a}}<\boldsymbol{1}$, implica que la frecuencia de fallas es mayor en el arranque bajo estudio que en la línea troncal, por lo tanto el arranque debe considerarse como una nueva línea troncal, tras la evaluación del costo-beneficio que esto implicará a la empresa distribuidora.

- Si $\boldsymbol{F} \boldsymbol{D}_{\boldsymbol{T a}}>\mathbf{1}$, implica que el arranque analizado no precisa de mayor inversión debido a que su peso en el índice de fallas es mínimo.

- $\quad$ Si se tiene presente el crecimiento de la densidad de demanda $\mathbf{D}\left(\mathrm{KVA} / \mathrm{km}^{2}\right)$ que alimenta el arranque $\boldsymbol{a}_{\boldsymbol{i}}$ bajo análisis, se establece arbitrariamente como criterio que para despreciar la influencia de fallas del arranque sobre la troncal $\boldsymbol{F} \boldsymbol{D}_{\boldsymbol{T a}}>\mathbf{0 . 8}$, lo que implicará soportar un aumento del $20 \%$ en la frecuencia de fallas, tras el aumento de la demanda.

\section{EFECTO DEL NÚMERO DE RECONECTADORES}

Según lo visto en el punto anterior, se estableció que el factor $F D_{T a}$ (Factor de distancia entre Troncal y Arranque), puede ser utilizado como estrategia de minimización del FMIK del alimentador bajo análisis. Otra forma de minimizar la frecuencia de fallas en un alimentador es la conexión a la línea de una mayor cantidad de equipos de protección (reconectadotes, por ejemplo) y/o equipos de maniobra (seccionadores, por ejemplo), los cuales permiten despejar las fallas y minimizar la cantidad de usuarios sin suministro. Esta es la estrategia más utilizada y en la cual se presentará un método de optimización lineal de ubicación.

Bajo lo planteado en (4) se considerarán reconectadores en la línea troncal, por tener el mayor aporte en el índice de frecuencia de fallas. Los reconectadores son equipos de protección que permiten la reposición del servicio sin necesidad de desconexiones operacionales, por lo que esta ventaja respecto de otras protecciones es tomada en cuenta en este trabajo. Al mismo tiempo los arranques de la troncal consideran protecciones del tipo fusible, lo que involucra una desconexión del arranque una vez operada la protección.

Considere el alimentador de figura 5, de longitud $\mathbf{L}(\mathrm{km})$ con $\mathbf{K V A}_{\mathrm{T}}$ carga total conocida y uniformemente distribuida a través de la línea. Esto significa que la función de carga acumulada $\Gamma(\mathbf{X})$ es una función lineal de la distancia medida desde la protección de la cabecera (tomando como referencia la fuente principal $\mathrm{Fte}_{1}$ ).

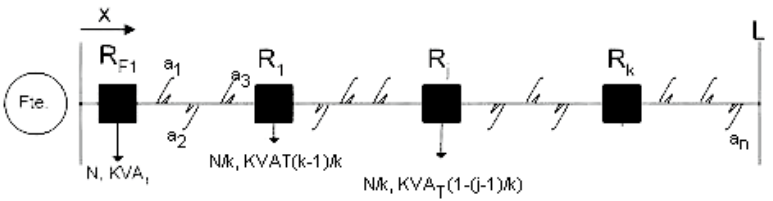

Figura 5. Fallas detectadas y carga KVA interrumpida por cada reconectador.

Tabla 2. Tabla de eventos para cálculo del índice de frecuencia.

\begin{tabular}{|c|c|c|c|}
\hline $\begin{array}{c}\text { Protec. } \\
\text { Operada }\end{array}$ & $\begin{array}{c}\text { Tasa de } \\
\text { Falla }\end{array}$ & KVA int. & $\begin{array}{c}\text { Frecuencia de } \\
\text { Interrupción } \mathbf{F}_{\mathbf{i}}\end{array}$ \\
\hline $\mathrm{R}_{\mathrm{F} 1}$ & $\lambda_{T} \cdot L / k$ & $K V A_{T}$ & $N$ \\
\hline $\mathrm{R}_{1}$ & $\lambda_{T} \cdot L / k$ & $K V A_{T}\left(1-\frac{1}{k}\right)$ & $\frac{N}{k} \cdot K V A_{T}\left(1-\frac{1}{k}\right)$ \\
\hline $\mathrm{R}_{\mathrm{j}}$ & $\lambda_{T} \cdot L / k$ & $K V A_{T}\left(1-\frac{j-1}{k}\right)$ & $\frac{N}{k} \cdot K V A_{T}\left(1-\frac{j-1}{k}\right)$ \\
\hline $\mathrm{a}_{1}$ & $\lambda_{a_{1}} \cdot b / 2$ & $K V A_{T} / n$ & $\frac{\lambda_{a 1} \cdot b \cdot n}{2} \cdot \frac{K V A_{T}}{n}$ \\
\hline $\mathrm{a}_{\mathrm{n}}$ & $\lambda_{a_{n}} \cdot b / 2$ & $K V A_{T} / n$ & $\frac{\lambda_{a_{n}} \cdot b}{2} \cdot \frac{K V A_{T}}{n}$ \\
\hline
\end{tabular}


Para simplicidad del análisis, considérese que las fallas se distribuyen uniformemente a lo largo del alimentador. Esto equivale asumir que la tasa de falla $\lambda_{T}$ es constante a lo largo de la línea. Sea $N=\lambda_{T} \cdot L$ el total de fallas, $\boldsymbol{k}$ el número de reconectadores igualmente espaciados, por lo que la tasa de falla entre reconectadores estará dada por $N_{k}=\lambda_{T} \cdot L / k$, donde además $\boldsymbol{R}_{j}$ corresponderá al j-ésimo reconectador a lo largo de la línea, $\boldsymbol{a}_{i}$ el i-ésimo arranque con un total de $\boldsymbol{n}$. Entonces, el número de fallas aisladas y la carga KVA despejada por cada equipo $\boldsymbol{R}_{\boldsymbol{j}}$ se deriva desde la tabla de eventos que se muestra en tabla 2 anterior.

FMIK de la línea mostrada en la figura 5 con $\left(\lambda_{\mathrm{T}}=\lambda_{a_{i}}=\lambda\right)$ :

$$
\begin{aligned}
& F M I K_{\text {Total }}=F M I K_{\text {Troncal }}+F M I K_{\text {Arranque }} \\
& F_{\text {TII }}=\sum_{j=0}^{k-1}\left(L \frac{(k-j)}{k^{2}}\right)+\frac{b}{2}
\end{aligned}
$$

La figura 6 muestra que $\mathbf{R}_{\mathbf{F 1}}$ detectará $\mathbf{N}$ fallas y provocará la desconexión del total de la carga conectada $\boldsymbol{K V} \boldsymbol{A}_{T}$. La protección $\mathbf{R}_{\mathbf{j}}$ detectará $\lambda_{T} \cdot L / k$ fallas, desconectando $K V A_{T}(\mathbf{1}-(j-1) / k)$ KVA de la troncal. Las ecuaciones (6) y (7) muestran cómo la adición de equipos de protección disminuye la frecuencia equivalente de interrupción en la troncal, minimizando así FMIK Total considerando $\lambda=\lambda_{T}=\lambda_{a}$.

$$
\lim _{k \rightarrow \infty} F M I K_{\text {Troncal }}=\lambda \cdot L \cdot \lim _{k \rightarrow \infty} \sum_{j=0}^{k-1}\left(\frac{(k-j)}{k^{2}}\right) \rightarrow 0
$$

Calculando $F D_{T a}(5)$ se obtiene:

$$
F D_{T a}=2 \frac{\lambda_{T}}{\lambda_{a}} \sum_{j=0}^{k-1}\left(\frac{k-j}{k^{2}}\right)^{\lambda_{T}=\lambda_{a}}=2 \sum_{j=0}^{k-1}\left(\frac{k-j}{k^{2}}\right)
$$

La expresión anterior (8) implica para el caso en estudio $\left(\lambda_{\mathrm{T}}=\lambda_{a_{i}}\right)$ que entre mayor número de reconectadores, mayor será la influencia en la frecuencia de falla de los arranques sobre la troncal, es decir, bajo una tasa de falla constante el $\boldsymbol{F} \boldsymbol{D}_{T a}$ permite discriminar la cantidad de reconectadores máximo en la troncal, de manera que la influencia de los arranques siga siendo menor a la influencia de la tasa de falla propia de la troncal.

\section{EFECTO DEL NÚMERO DE FUENTES}

Bajo las especificaciones del esquema presentado en la figura 6 , y considerando un equipo de protección y/o maniobra en estado NA en la línea troncal, el cual tiene un tiempo de respuesta nulo (instantáneo) y que permite el ingreso de una segunda fuente independiente sincronizada a la red (supuesta sin límite de capacidad, 100\% disponible, $100 \%$ confiable, al igual que para el interruptor).

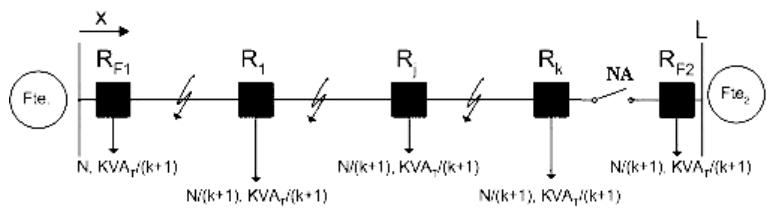

Figura 6. Sistema considerando Troncal con presencia de interruptor NA.

Analizando la línea desde la $\mathrm{Fte}_{1}$ y considerando la independencia de las fuentes presentes, entonces es posible hacer el análisis de carga interrumpida desde $\mathrm{Fte}_{1}$ ó $\mathrm{Fte}_{2}$, esto implica que los reconectadores serán elementos de aislamiento de fallas (suponiendo sólo una falla a la vez). Por lo que al existir una falla entre los reconectadores $R_{i}$ y $R_{j}$, ésta quedará aislada y el resto del circuito será alimentado con $\mathrm{Fte}_{2}$ cerrando el equipo NA, tal como se muestra en la figura 6.

La tabla 3 muestra la secuencia de eventos que permite calcular los índices de frecuencias considerando la fuente principal Fte 1 y el equipo de protección y/o maniobra NA que permite la alimentación desde $\mathrm{Fte}_{2}$.

Tabla 3. Tabla de eventos para cálculo del índice de frecuencia considerando $\mathrm{Fte}_{1}$ e interruptor NA

\begin{tabular}{|l|l|c|c|}
\hline $\begin{array}{c}\text { Fuente en } \\
\text { Operación }\end{array}$ & $\begin{array}{c}\text { Protección } \\
\text { Operada }\end{array}$ & $\begin{array}{c}\text { Tasa de } \\
\text { Falla }\end{array}$ & $\begin{array}{c}\text { KVA } \\
\text { interrumpidos }\end{array}$ \\
\hline $\mathrm{F} 1$ & $\mathrm{R}_{\mathrm{F} 1}$ & $\lambda \cdot L /(k+1)$ & $K V A_{T} / k+1$ \\
\hline & $\mathrm{R}_{1}$ & $\lambda \cdot L /(k+1)$ & $K V A_{T} / k+1$ \\
\hline & $\mathrm{R}_{\mathrm{n}}$ & $\lambda_{T} \cdot L /(k+1)$ & $K V A_{T} / k+1$ \\
\hline $\mathrm{F} 2$ & $\mathrm{R}_{\mathrm{F} 2}$ & $\lambda_{T} \cdot L /(k+1)$ & $K V A_{T} / k+1$ \\
\hline & $\mathrm{R}_{\mathrm{n}}$ & $\lambda_{T} \cdot L /(k+1)$ & $K V A_{T} / k+1$ \\
\hline & $\mathrm{R}_{\mathrm{n}-1}$ & $\lambda_{T} \cdot L /(k+1)$ & $K V A_{T} / k+1$ \\
\hline & $\mathrm{R}_{1}$ & $\lambda_{T} \cdot L /(k+1)$ & $K V A_{T} / k+1$ \\
\hline Fusibles & $\mathrm{F}_{\mathrm{i}}$ & $\lambda_{a_{i}} b / 2$ & $\frac{K V A_{T}}{(k+1) \cdot n}$ \\
\hline
\end{tabular}




$$
\begin{aligned}
F M I K & =\sum \frac{K V A_{\text {int }}}{K V A_{T}} \quad, \lambda_{T}=\lambda_{a_{i}}=\lambda \\
& =\sum_{j=1}^{2 \cdot k} \frac{\lambda \cdot L}{(k+1)^{2}}+\frac{\lambda \cdot b}{2} \\
& =2 \cdot k \frac{\lambda \cdot L}{(k+1)^{2}}+\frac{\lambda \cdot b}{2}
\end{aligned}
$$

Como era de esperar (9) y (10) muestran que la adición de equipos de protección disminuye la frecuencia equivalente de interrupción en la troncal, minimizando FMIK $_{\text {Total }}$.

$$
\lim _{k \rightarrow \infty} F_{\text {Troncal }}=\lambda \cdot L \cdot \lim _{k \rightarrow \infty} \sum_{j=1}^{2 k}\left(\frac{1}{(k+1)^{2}}\right) \rightarrow 0
$$

Calculando $F D_{T a}(5)$ se obtiene:

$$
F D_{T a}=\sum_{j=1}^{2 k}\left(\frac{2}{(k+1)^{2}}\right)
$$

Debido a la presencia de una segunda fuente $\mathrm{Fte}_{2}$ se disminuye al doble la influencia de las fallas de los arranques sobre la troncal. Esto puede extenderse a futuros estudios en la integración de generación distribuida y analizar la frecuencia de fallas en la troncal aumentando el número de reconectadores.

\section{UBICACIÓN ÓPTIMA DE RECONECTADORES}

Para determinar la ubicación óptima de los reconectadores, se utilizará el método presentado en [3], de forma de minimizar el índice FMIK. Para ello se expresarán los $\mathrm{KVA}_{\text {int }}$ en función de la posición de los equipos presentes en la troncal. Los reconectadores en la troncal se conectarán como se muestra en figura 7. El índice de frecuencia resultará una función de $\mathbf{n}-\mathbf{r}_{\mathbf{c a b}}$ variables, donde $\mathbf{n}$ representa los $\boldsymbol{n}$ reconectadores a lo largo de la troncal y $\mathbf{r}_{\text {cab }}$ los $\boldsymbol{r}$ reconectadores de cabecera presentes a lo largo de las troncales. Para el caso según tabla 2, el problema se reducirá a n-1 variables y para Tabla 3, el problema a resolver corresponderá a n-2 variables. Por ejemplo: Si tres equipos fueran conectados a lo largo de la troncal cuando consideramos además la presencia de un equipo en estado NA, el problema se reduce a encontrar las posiciones $\mathbf{x}, \mathbf{y}, \mathbf{z}$ de cada uno a lo largo de la troncal, medidas desde la fuente principal; esto implicará minimizar la frecuencia de fallas, es decir, encontrar el $\min \left(\Sigma F_{i}\right)=\min \left(\Sigma E_{\text {ventos }} \cdot K V A_{\text {int }}(x, y, z)\right)$
La figura 7 presenta un alimentador con 2 reconectadores instalados en $\mathbf{x}$ e $\mathbf{y}(\mathrm{km})$ a lo largo de la troncal. Sea $\Gamma(\mathrm{X})$ la función de carga KVA acumulada a lo largo de la línea desde $\mathrm{R}_{\mathrm{F} 1}$, entonces $\Gamma(\mathrm{L})=\mathrm{KVA}_{\mathrm{Total}}$. Además, considere que $F(X)=\lambda \cdot X$ es la función de fallas acumuladas a lo largo de la línea, desde $\mathrm{R}_{\mathrm{F} 1}$. Luego $\mathrm{F}(\mathrm{L})=\mathrm{N}$ el total de fallas que afecta al alimentador. Para obtener las expresiones analíticas de las funciones $\mathrm{F}(\mathrm{X})$ y $\Gamma(\mathrm{X})$ debe recurrirse a la data de fallas y de KVA conectados a lo largo de las líneas. En tabla 4 se presenta la evolución sin considerar la presencia de equipo NA y la tabla 5 considera la presencia del NA y por ende ambas fuentes, en función de $\mathrm{F}(\mathrm{X})$ y $\Gamma(\mathrm{X})$.

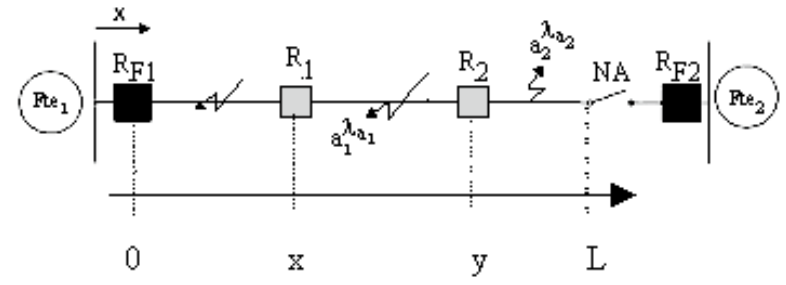

Figura 7. Alimentador de largo L (km) con reconectadores.

$\mathrm{El}$ análisis considera que $\mathrm{Fte}_{1}$ es la fuente principal y el origen del alimentador, entonces las condiciones de borde del problema indican que $\boldsymbol{x}>\boldsymbol{0}$ e $\boldsymbol{y}>\boldsymbol{x}$. Cabe mencionar que en presencia de dos fuentes la línea se comporta redundante desde el punto de vista de análisis de falla, por lo que el aporte en las condiciones de falla se traduce en la suma algebraica del aporte de cada una.

\section{EJEMPLO DE APLICACIÓN}

Se aplica el método a un alimentador ficticio de largo 25,3 $(\mathrm{km})$ y de carga mostrada en figura 7. La función de fallas acumuladas $\mathrm{F}(\mathrm{X})$ se supuso lineal $\left(\lambda_{\mathrm{T}}=0.227\right.$; constante). Luego:

$$
F(X)=0,227 \cdot x
$$

Donde $\mathbf{x}$ es la distancia en $\mathrm{km}$ medida desde la cabecera del alimentador visto desde el alimentador principal Fte ${ }_{1}$.

Tabla 4. Tabla de eventos en términos de $\mathrm{F}(\mathrm{x})$ y $\Gamma(\mathrm{x})$ $\sin$ NA.

\begin{tabular}{|l|l|l|l|}
\hline \# eventos & \multicolumn{1}{|c|}{$\mathbf{R}$} & \multicolumn{1}{|c|}{ KVA $_{\text {int }}$} & \multicolumn{1}{c|}{$\begin{array}{c}\text { Frecuencia de } \\
\text { Interrupción } \mathbf{F}_{\mathbf{i}}\end{array}$} \\
\hline $\mathrm{F}(\mathrm{x})$ & $\mathrm{R}_{\mathrm{F} 1}$ & $\Gamma(\mathrm{L})=\mathrm{KVA}_{\mathrm{T}}$ & $F(x) \cdot \Gamma(L)$ \\
\hline $\mathrm{F}(\mathrm{y})-\mathrm{F}(\mathrm{x})$ & $\mathrm{R}_{2}$ & $\Gamma(\mathrm{L})-\Gamma(\mathrm{x})$ & {$[F(y)-F(x)] \cdot[\Gamma(L)-\Gamma(x)]$} \\
\hline $\mathrm{F}(\mathrm{L})-\mathrm{F}(\mathrm{y})$ & $\mathrm{R}_{3}$ & $\Gamma(\mathrm{L})-\Gamma(\mathrm{y})$ & {$[F(L)-F(y)] \cdot[\Gamma(L)-\Gamma(y)]$} \\
\hline
\end{tabular}


Tabla 5. Tabla de Eventos en términos de $\mathrm{F}(\mathrm{x})$ y $\Gamma(\mathrm{x})$ con NA.

\begin{tabular}{|l|l|l|l|l|}
\hline Fuente & \# eventos & $\mathbf{R}$ & KVA $_{\text {int }}$ & \#eventos $^{*} \mathbf{K V A}_{\text {int }}$ \\
\hline $\mathrm{Fte}_{1}$ & $\mathrm{~F}(\mathrm{x})$ & $\mathrm{R}_{\mathrm{F} 1}$ & $\Gamma(\mathrm{x})$ & $F(x) \cdot \Gamma(x)$ \\
\hline & $\mathrm{F}(\mathrm{y})-\mathrm{F}(\mathrm{x})$ & $\mathrm{R}_{1}$ & $\Gamma(\mathrm{y})-\Gamma(\mathrm{x})$ & {$[F(y)-F(x)] \cdot[\Gamma(y)-\Gamma(x)]$} \\
\hline & $\mathrm{F}(\mathrm{L})-\mathrm{F}(\mathrm{y})$ & $\mathrm{R}_{2}$ & $\mathrm{KVA}_{\mathrm{T}}-\Gamma(\mathrm{y})$ & {$[F(L)-F(y)] \cdot[\Gamma(L)-\Gamma(y)]$} \\
\hline $\mathrm{Fte}_{2}$ & $\mathrm{~F}(\mathrm{~L})-\mathrm{F}(\mathrm{y})$ & $\mathrm{R}_{\mathrm{F} 2}$ & $\mathrm{KVA}_{\mathrm{T}}-\Gamma(\mathrm{y})$ & {$[F(L)-F(y)] \cdot[\Gamma(L)-\Gamma(y)]$} \\
\hline & $\mathrm{F}(\mathrm{y})-\mathrm{F}(\mathrm{x})$ & $\mathrm{R}_{2}$ & $\Gamma(\mathrm{y})-\Gamma(\mathrm{x})$ & {$[F(y)-F(x)] \cdot[\Gamma(y)-\Gamma(x)]$} \\
\hline & $\mathrm{F}(\mathrm{x})$ & $\mathrm{R}_{1}$ & $\Gamma(\mathrm{x})$ & $F(x) \cdot \Gamma(x)$ \\
\hline
\end{tabular}

Entonces la frecuencia de interrupción total:

$$
\begin{aligned}
& N \cdot Q_{T}-\Gamma(x) \cdot F(y)+\Gamma(x) \cdot F(x) \\
& +\Gamma(y) \cdot F(y)-N \cdot \Gamma(y)
\end{aligned}
$$

Con lo cual la frecuencia de interrupción total es:

$$
2 \cdot\left[\begin{array}{l}
2 \cdot F(x) \cdot F(y)-F(x) \cdot \Gamma(y)+2 \cdot F(y) \cdot \Gamma(y) \\
-F(y) \cdot \Gamma(x)-\Gamma(y) \cdot N-F(y) \cdot K V A_{T}+N \cdot K V A_{T}
\end{array}\right]
$$

La figura 8 muestra el gráfico correspondiente, el modelo adoptado y su coeficiente de correlación R. Luego la función polinómica de aproximación de $\Gamma(\mathrm{x})$ apropiada es:

$$
\begin{aligned}
\Gamma(x) & =-3 \cdot 10^{-5} x^{5}+1 \cdot 10^{-4} x^{4}+0,5095 x^{3}-7,1879 x^{2} \\
& +66,858 x-18,259
\end{aligned}
$$

En base a esta información se reconstruyen las tablas 4 y

\begin{tabular}{|c|c|c|c|c|c|}
\hline \multirow{2}{*}{$\begin{array}{c}\text { Ftes } \\
\text { Presentes }\end{array}$} & \multicolumn{2}{|c|}{$\mathbf{N}^{\circ}$ Reconect. } & \multirow{2}{*}{$\begin{array}{l}\text { x opt. } \\
(\mathbf{k m})\end{array}$} & \multirow{2}{*}{$\begin{array}{c}\text { y opt. } \\
\text { (km) }\end{array}$} & \multirow{2}{*}{ FMIK } \\
\hline & Total & Troncal & & & \\
\hline Fte1 $\sin$ NA & 2 & 1 & 17.2 & - & 5.07 \\
\hline Fte1 $\sin \mathrm{NA}$ & 3 & 2 & 12.9 & 19.6 & 4.31 \\
\hline Fte 1 con NA & 3 & 1 & 15.1 & - & 5.3 \\
\hline Fte $1 \operatorname{con} \mathrm{NA}$ & 4 & 2 & 11.5 & 18.7 & $* 3.47$ \\
\hline
\end{tabular}
5 obteniéndose los resultados presentados en la tabla 6 .

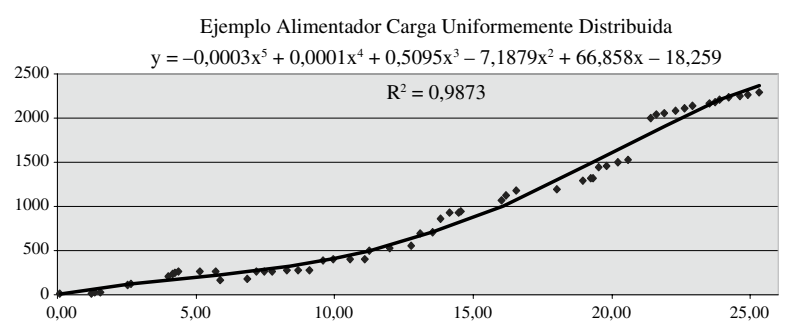

Figura 8. Función de carga acumulada $\Gamma(\mathrm{X})$.

Tabla 6. Ubicación óptima de reconectadores.
*Al incluir el NA suman 4 reconectadores, de acuerdo a la figura 7 disminuyendo el FMIK al optimizar la ubicación de los reconectadores. Considerando los arranques $\mathrm{a}_{1}{ }^{\lambda a l}=0.1$ y el arranque $\mathrm{a}_{2}{ }^{\lambda a 2=0.21}$ y ambos arranques de largo $\mathrm{b}=5$

\begin{tabular}{|c|c|c|c|c|c|}
\hline \multirow{3}{*}{$\begin{array}{c}\text { Ftes } \\
\text { Presentes } \\
\text { Fte1 } \sin \text { NA }\end{array}$} & \multicolumn{2}{|c|}{$\mathbf{N}^{\circ}$ Reconect. } & \multirow{3}{*}{$\begin{array}{c}\text { Tasa Falla } \\
\mathrm{A}_{1}^{\lambda a 1=0.1}\end{array}$} & \multirow{2}{*}{\multicolumn{2}{|c|}{$\mathbf{F D}_{\mathrm{TA}}$}} \\
\hline & \multirow{2}{*}{\begin{tabular}{|c|} 
Total \\
3 \\
\end{tabular}} & \multirow{2}{*}{$\begin{array}{c}\text { Troncal } \\
2\end{array}$} & & & \\
\hline & & & & 3.41 & (8) \\
\hline Ftel con NA & 4 & 2 & $\mathrm{~A}_{1}^{\lambda a 1=0.1}$ & 2.01 & (11) \\
\hline Fte1 $\sin$ NA & 3 & 2 & $A_{2}^{\lambda a 2=0.21}$ & 1.62 & (8) \\
\hline Fte1 con NA & 4 & 2 & $A_{2}^{\lambda a 2=0.21}$ & $* * 0.96$ & (11) \\
\hline
\end{tabular}
$\mathrm{km}$. Se construye la tabla 7 que indica el $\mathrm{FD}_{\mathrm{TA}}$ de ellos de acuerdo a (11) y (8).

Tabla 7. Incidencia de arranques $\mathrm{a}_{1} \mathrm{y} \mathrm{a}_{2}$ en índice $\mathrm{FD}_{\mathrm{TA}}$

**Ante la presencia del NA, el arranque $\mathrm{a}_{2}$ entra en el rango menor a 1 , lo que equivale a estudiarlo como un nuevo alimentador: Esto se explica, en que la introducción de dos reconectadores en la troncal con NA minimizó el FMIK y llevó a que $\mathrm{a}_{2}$ tuviera mayor incidencia que cuando estaba en presencia sólo de Fte ${ }_{1}$. Esto deja ver que el número de reconectadores en la línea troncal influye en el peso que toman los arranques sobre la misma, por lo que es necesario un análisis técnico económico entre la cantidad de reconectadores a utilizar y la transformación de arranques importantes $\left(\mathrm{FD}_{\mathrm{TA}}<1\right)$ en nuevas líneas troncales, de forma de llevar el FMIK a un valor mínimo.

\section{CONCLUSIONES}

En este trabajo se introdujo un nuevo índice $\mathrm{FD}_{\mathrm{TA}}$ el cual permite evaluar la incidencia que pueden tener los arranques sobre una línea troncal con carga uniformemente distribuida, esto en función de un número $k$ de reconectadores, presentes (o no) en la línea, que minimizan el índice de frecuencias de interrupción y por ende el índice FMIK. Esta metodología permite establecer la cantidad máxima de reconectadores, de forma tal que la incidencia de los arranques no sobrepase a la línea troncal, implicando con ello una importante limitante y restricción sobre las inversiones a realizar por la empresa distribuidora. Cabe destacar que la inversión no tan sólo involucra la introducción de nuevos equipos de maniobra, sino también el estudio de los arranques que bajo su data histórica de falla y el $\mathrm{FD}_{\mathrm{Ta}}$ establecen un criterio de selección para creación de nuevos alimentadores, optimizando a su vez el índice FMIK. 
Los resultados del ejemplo propuesto muestran la bondad de la metodología de optimización en presencia de NA, implicando con ello restauración del servicio y minimizar el FMIK.

\section{AGRADECIMIENTOS}

Este trabajo se realizó con el financiamiento de la Dirección de Investigación de la Universidad de Concepción, Chile en el proyecto DIUC 207.092.048-1.0 Además, los autores desean agradecer al Ingeniero Sr. Gabriel Olguín, por su valiosa colaboración y comentarios durante la elaboración de este trabajo.

\section{REFERENCIAS}

[1] Reglamento de la ley General de Servicios Eléctricos. Decreto Supremo 327. República de Chile. 1998. Actualizado 2006.

[2] A. Cárcamo, L. García y J. Pezoa. "Reconfiguración de Redes Eléctricas de Media Tensión basada en el Algoritmo de PRIM". Ingeniare. Revista chilena de ingeniería. Vol. $15 \mathrm{~N}^{\mathrm{o}}$ 1, pp. 83-91. Abril 2007.

[3] A. Cárcamo-Gallardo, L. García-Santander and J.E. Pezoa. "Greedy reconfiguration algorithms for medium-voltage distribution networks". IEEE Trans. Power Delivery. Vol. 24, pp. 328-337. 2009.

[4] L. García, H. Opazo and E. López. "Switches optimum location in medium voltage network via minimization of energy not supplied". Proc. IEEE Computers Society, 16th International Conference on Elect., Communications and Computers. Universidad de las Américas. Puebla, México. Febrero 2006.
[5] W. Min and H Chan. "A new approach for distribution feeder reconfiguration for loss reduction and service restoration". IEEE Trans. Power Delivery. Vol. 13, pp. 870-875. 1998.

[6] Comisión Nacional de Energía. CNE "Norma Técnica de Seguridad y Calidad de Servicio". Mayo 2005.

[7] Ranking Calidad de Servicio de empresas distribuidoras de energía en Chile. URLs: www. sec.cl. Fecha de consulta: Abril 2008.

[8] IEEE Std. 1366 ${ }^{\mathrm{TM}}$ 2003. Guide for Electric Power Distribution Reliability Indices. IEEE Power Engineering Society.

[9] Nagaraj Balijepalli, Subrahmanyam S. Venkata and Richard D. Christie. "Predicting Distribution System Perfomance Against Regulatory Reliability Standards". IEEE Trans. On Power Delivery. Vol. 19 $\mathrm{N}^{\circ} 1$, pp. 350-356. January 2004.

[10] José Riquelme Pérez y Luis García-Santander. "Emplazamiento óptimo de equipos de protección y/o maniobra considerando variabilidad de la demanda". Tesis para optar al título de Ingeniero Civil Eléctrico. Departamento de Ingeniería Eléctrica. Universidad de Concepción. Agosto 2008.

[11] G. Ricardo Baeza, P. José Rodríguez, J.L. Hernández S. "Evaluación de confiabilidad de sistemas de distribución eléctrica en desregulación”. Ingeniare. Revista chilena de ingeniería. Vol. 11 N 1, pp. 33-39. 2003.

[12] Yang Wenyu, Liu Jian, Yu Jianmin, Dong Haipeng and Song Meng. "Optimal allocation of switches in dsitribution Networks". Proc. of the 5th word Congress on Intelligent Control and Automation, pp. 5155-5159. Hanzhou, PR. China. June 15-19, 2004. 